\title{
Radical Cyclopropanol Ring Opening Initiated Tandem Cycliza- tions for Efficient Synthesis of Phenanthridines and Oxindoles
}

Dexter C. Davis

Christopher W. Haskins

Mingji Dai*

Synlett 2017, 28, 913.

In Scheme 1 of the e-first version of this article intermediate $\mathbf{B}$ contained one extra " $\mathrm{H}$ " on to the carbon bearing the radical. It was added inadvertently during the generation of the final graphic file. This structure was corrected for the final online and print versions. The editorial office apologizes for the error. 